\title{
Lactancia materna exclusiva prolongada: ¿contribuye a la anemia en niños menores de 5 años de hogares indígenas del Perú?
}

Continued breastfeeding may contribute to anemia in Peruvian children under five of indigenous households

\author{
Eddy López Huamanrayme ${ }^{1,2, a}$, Noé Atamari-Anahui ${ }^{1,2, a}$, Abel Mestas-Valero ${ }^{1,3, b}$ \\ 'Escuela de Medicina, Universidad Nacional San Antonio Abad del Cusco, Cusco, Perú. \\ ${ }^{2}$ Asociación Científica de Estudiantes de Medicina Humana del Cusco (ASOCIEMH-CUSCO), Cusco, Perú. \\ ${ }^{3}$ Dirección Regional de Salud del Cusco (DIRESA-CUSCO), Cusco, Perú. \\ a Estudiante de medicina; ${ }^{b}$ Doctor en salud pública.
}

An Fac med. 2016;77(1):67 / http://dx.doi.org/10.15381/anales.v77i1.11558

\section{SR. EDITOR:}

Leímos con mucho interés el artículo publicado por Flores-Bendezú y col ${ }^{(1)}$, donde se menciona que la prevalencia de anemia en niños menores de 5 años de hogares indígenas fue de $47,5 \%$, resaltando que la totalidad de niños menores de 12 meses presentaba anemia, considerando como posibles causas el deficiente consumo de alimentos ricos en hierro y la enteroparasitosis ${ }^{(1)}$.

La lactancia materna exclusiva (LME) más de 6 meses o inicio tardío de la ablactancia podría ser también considerado un factor contribuyente a la anemia. Pasricha y col. (2), en su estudio realizado en zonas rurales de la India que incluyó a 405 niños (edad, 12 a 23 meses) de nivel socioeconómico bajo, encontraron una prevalencia de anemia cerca de 75,3\%, donde la anemia estaba directamente asociada a la continuidad de la lactancia materna más de 6 meses. De igual forma, Marques y col. ${ }^{(3)}$, en su estudio realizado en Brasil y que incluyó a 102 niños, demostraron que la lactancia materna exclusiva fue un factor protector de anemia solo hasta los 4 meses y que posterior a ese tiempo incrementaba su riesgo. Los antecedentes mencionados evidencian una relación entre anemia y LME más allá de los 6 meses, que sustenta- ría algunos resultados encontrados por Flores-Bendezú y col ${ }^{(1)}$, lo cual consideramos que debería ser mencionado, puesto que en la Encuesta Demográfica y de Salud Familiar (ENDES) 2013 se señala que la LME es más duradera en la selva, especialmente en áreas rurales en comparación con la costa, pero inferior a la de la sierra.

Consideramos oportuno realizar estudios para ver la relación de anemia y la lactancia materna exclusiva prolongada en niños de poblaciones rurales de la selva y sierra del Perú, las cuales vienen siendo las más vulnerables frente a este problema ${ }^{(4,5)}$. De esta manera se aportaría al desarrollo y mejora de estrategias e intervenciones nacionales para disminuir la prevalencia de anemia en niños ${ }^{(6)}$, puesto que aún sigue siendo un problema de salud pública en nuestro país ${ }^{(7)}$.

\section{REFERENCIAS BIBLIOGRÁFICAS}

1. Flores-Bendezú J, Calderón J, Rojas B, AlarcónMatutti E, Gutiérrez C. Desnutrición crónica y anemia en niños menores de 5 años de hogares indigenas del Perú: análisis de la encuesta demográfica y de salud familiar 2013. An Fac med. 2015;76(2):135-40.

2. Pasricha S-R, Black J, Muthayya S, Shet A, Bhat $V$, Nagaraj $S$, et al. Determinants of anemia among young children in rural India. Pediatrics. 2010;126(1):e140-9. doi: 10.1542/peds.2009-3108.
3. Marques RFSV, Taddei JAAC, Lopez FA, Braga JAP, Marques RFSV, Taddei JAAC, et al. Breastfeeding exclusively and iron deficiency anemia during the first 6 months of age. Rev Assoc Médica Bras. 2014;60(1):18-22.

4. Anticona C, San Sebastian M. Anemia and malnutrition in indigenous children and adolescents of the Peruvian Amazon in a context of lead exposure: a cross-sectional study. Glob Health Action. 2014;7:22888. doi: 10.3402/gha.v7.22888.

5. Gómez-Guizado G, Munares-Garcia O. Anemia y estado nutricional en lactantes de dos a cinco meses atendidos en establecimientos del Ministerio de Salud del Perú, 2012. Rev Peru Med Exp Salud Publica. 2014;31(3):487-93.

6. Huamán-Espino L, Aparco JP, Nuñez-Robles E, Gonzáles E, Pillaca J, Mayta-Tristán P. Consumo de suplementos con multimicronutrientes Chispitas ${ }^{\circ}$ y anemia en niños de 6 a 35 meses: estudio transversal en el contexto de una intervención poblacional en Apurimac, Perú. Rev Peru Med Exp Salud Publica. 2012;29(3):314-23.

7. Mujica-Coopman MF, Brito A, López de Romaña D, Ríos-Castillo I, Coris H, Olivares M. Prevalence of anemia in Latin America and the Caribbean. Food Nutr Bull. 2015;36 Suppl 2:S119-28.

Carta al Editor recibida el 3 de octubre de 2015.

Fuentes de financiamiento: Autofinanciado.

Conflictos de interés: Los autores declaran no tener conflictos de interés.

Correspondencia:

Eddy López Huamanrayme.

Dirección: Sol de América G-12. San Sebastián, Cusco, Perú.

Teléfono: (084)973189924

Correo electrónico: eddylopez.unsaac@gmail.com 